\title{
The Hotspots of Sports Science and the Effects of Knowledge Network on Scientific Performance Based on Bibliometrics and Social Network Analysis
}

\author{
Linxiao Ma $\mathbb{D}^{1},{ }^{1}$ Yuzhu Wang $\mathbb{D},{ }^{2}$ Yue Wang $\mathbb{D},{ }^{3}$ Ning Li $\mathbb{D},{ }^{3}$ Sai-Fu Fung $\mathbb{D}^{4},{ }^{4}$ Lu Zhang $\mathbb{D}^{3},{ }^{3}$ \\ and Qian Zheng ${ }^{3}{ }^{3}$
}

\author{
${ }^{1}$ Sport College, Xi'an University of Architecture and Technology, Xi'an 710311, China \\ ${ }^{2}$ Shandong Sport University, Jinan 250063, China \\ ${ }^{3}$ Xi'an Physical Education University, Xi'an 710065, China \\ ${ }^{4}$ City University of Hong Kong, Kowloon Tong, Hong Kong
}

Correspondence should be addressed to Yuzhu Wang; wangyuzhu@sdpei.edu.cn

Received 18 March 2021; Revised 17 April 2021; Accepted 8 May 2021; Published 24 May 2021

Academic Editor: Fei Xiong

Copyright $\odot 2021$ Linxiao Ma et al. This is an open access article distributed under the Creative Commons Attribution License, which permits unrestricted use, distribution, and reproduction in any medium, provided the original work is properly cited.

In this study, we sorted out the research hotspots in sports science by bibliometric method and also used social network analysis to explore the relationship between knowledge networks and their scientific performance. We found 38 high-frequency keywords with obvious curricular nature or classical direction of sports science research and 4 high-frequency research groups. The topics of hotspots covered the secondary disciplines of sports science: physical education and training, national traditional sports, sports human science, and sports humanities and sociology. However, sports human science research is less; therefore, accelerating the research of sports human science is the focus of future research. Meanwhile, we use social network structure analysis (i.e., centrality, clustering coefficient, PageRank, and structural holes) to study the relationship between knowledge elements in knowledge networks and their scientific performance. In addition to betweenness centrality, the closeness centrality, clustering coefficient, and structural holes of knowledge elements are significantly and positively related to their influence. In the relationship between knowledge elements and productivity, betweenness centrality and closeness centrality show significant positive correlations, and clustering coefficient and structural hole show significant negative correlations. Therefore, knowledge networks can be used to predict the scientific performance of knowledge elements.

\section{Introduction}

In the context of the big data era, human beings produce large-scale behavioral data, and the development of computer technology enables the generated data to be stored [1]. By collecting, cleaning, and mining data to reveal the characteristics contained in the data, we can better understand human behavior and social interaction and provide new perspectives and methods for sociological research. With the rapid growth in the number of academic achievements of researchers, it is difficult for researchers to explore the research hotspots in their subject areas and the mechanisms underlying the impact of knowledge themes, and knowledge graph is a bibliometric visualization method developed on the basis of social network theory. It combines knowledge and methods from disciplines such as graph theory and information visualization techniques. It is able to show the knowledge development process and structural relationships and helps to understand the research hotspots and status quo of subject areas, etc. [2,3]. For the investigation of the intrinsic mechanisms of the influence of knowledge topics, social networks provide a good research perspective, which helps us to understand the strengths and weaknesses of the scientific research performance of knowledge elements by network features. 


\section{Data Collection and Measure}

2.1. Data Collection. According to the Annual Report on the Impact Factor of Chinese Academic Journals (2020 Edition), developed and published by the China Research Center for Scientific Bibliometric Evaluation and Tsinghua University Library, ten journals entered Q1 area. They were "Sports Science," "Journal of Beijing Sport University," "Journal of Shanghai Sport Institute," "Journal of Wuhan Sport Institute," "Sports Journal," "China Sports Science and Technology," "Sports and Science," "Sports Science Research," "Journal of Chengdu Sport Institute," and "Sports Culture Guide.” In 2018, Journal of Nanjing Sport Institute (Social Science) was renamed "Sports Science Research." Its paper had been published only for three years, so the data of "Sports Science Research" were discarded. In this paper, nine journals were selected from 2000 to 2020 and the paper type was academic journals, and the search time was December 11,2020 . There were 45,472 journal papers that met the criteria, and 43465 papers remained after excluding those with empty keywords. We filtered papers with 0 citations when examining the impact of knowledge networks on scientific performance.

\subsection{Measure}

2.2.1. Measure High-Frequency Keywords. Paper keywords are the summary of the paper content, which can express the research content of the paper more accurately. Through the statistics of the frequency of the occurrence of paper keywords, it can reveal the research hotspots and evolution trends in the subject field. Chu reveals that the research hotspots in the field of knowledge management in the recent ten years focus on knowledge management, knowledge sharing, tacit knowledge, library, knowledge management, systematic knowledge, knowledge economy, enterprise, knowledge transfer, knowledge service, explicit knowledge, and knowledge map through the word frequency statistics of paper keywords [4]. Li and Jiang analyzed the hot research topics of sports science in the recent five years, including competitive sports, sports management, mass sports, school sports, physical education, sports culture, sports history, traditional national sports, sports economy, and sports industry [5]. Jiang et al. conducted keyword analysis based on CSSCI database of sports humanities and sociology and found that the research hotspots of sports humanities and social sciences in the past five years were sports teaching, sports culture, competitive sports, sports industry and Olympics, with a decreasing trend in sports teaching research and an increasing trend in sports culture research [6]. This provides a reference for sports humanities and social science researchers.

Donohue proposed a method to distinguish between high- and low-frequency keywords [7]. The dividing line between high- and low-frequency keyword frequencies is defined as follows:

$$
\mathrm{TF}=\frac{-1+\sqrt{1+8 \mathrm{TF}_{1}}}{2},
$$

where TF refers to term frequency and $\mathrm{TF}_{1}$ is the number of keywords with a term frequency of 1 .

2.2.2. Measuring Knowledge Network Features. Social network analysis, also known as structural analysis, is a set of norms and methods to analyze the relational structure of social networks and their attributes [8-10], which helps us to measure the importance of nodes using their positional attributes. Common measures are centrality, clustering coefficient, and structural holes [11-15]. Abbasi et al. found a significant correlation between the attributes of authors in collaborative networks and $g$-index [11]. The papers are sorted in descending order by the number of citations, and when the cumulative number of citations is equal to the square of the order number, the order number is the $g$-index [15]. Yan and Ding found that the centrality of authors in coauthorship networks was significantly correlated with citation counts [13]. Guan et al. demonstrated that structural holes in knowledge networks are positively related to citation, and centrality has an inverted U-shaped relationship with citation, but they did not focus on the impact of knowledge networks on productivity [14]. Network features and performance studies mostly focus on co-authorship networks [11-13, 16-18], and fewer studies involve knowledge networks [14], and the measurement metrics are not comprehensive enough. In this paper, we use the centrality, clustering coefficients, and structural holes of knowledge network elements to explore their relationship with scientific research performance (productivity and impact).

\section{(1) Centrality}

(1) Degree centrality

Degree centrality is defined as the number of nodes that are directly connected to a node [11]. For node $i$ in the network, its degree centrality is calculated as follows:

$$
\mathrm{DC}_{i}=\sum_{j=1}^{n} x_{i j}(i \neq j),
$$

$\mathrm{DC}_{\mathrm{i}}$ is the degree of node $i, n$ is the number of nodes in the network, and $j$ is all nodes in the network except node $i$. When $i$ is adjacent to $j, x_{i j}=1$, and when $i$ is not adjacent to $j, x_{i j}=0$.

(2) Betweenness centrality

The idea of betweenness centrality: if a node is located on multiple shortest paths of other nodes, then the node is at the core of the network and has a large betweenness centrality [11]. For node $i$ in the network, its betweenness centrality is calculated as follows: 


$$
\mathrm{BC}_{i}=\sum \frac{d_{m n}(i)}{d_{m n}},
$$

$\mathrm{BC}_{i}$ is the betweenness centrality of node $i, d_{m n}(i)$ is the number of shortest paths of node $m$ and node $n$ through node $i$, and $d_{m n}$ is the number of shortest paths between node $m$ and node $n$.

(3) Closeness centrality

Closeness centrality is the inverse of the cumulative shortest path distance from a node to all other nodes [11], which is calculated as follows:

$$
C C_{i}=\sum_{j=1}^{n} \frac{1}{d(i, j)} \quad(i \neq j),
$$

$\mathrm{CC}_{i}$ is the closeness centrality of node $i, \mathrm{n}$ is the number of nodes in the network, $j$ is all the nodes in the network except node $i$, and $d(i, j)$ is the distance between node $i$ and node $j$.

(2) Clustering Coefficient. Clustering coefficient is a coefficient used to describe the degree of clustering between nodes in a graph, i.e., the degree of interconnection between neighbors of a node. The clustering coefficient is divided into global clustering coefficient and local clustering coefficient [19]. In this paper, we study the clustering coefficient of nodes, i.e., local clustering coefficient. For an undirected graph $G=(V, E), V$ is the set of nodes and $E$ is the set of edges. For node $i$, define the set of neighboring nodes as $N_{i}$, then the clustering coefficient of node $i$ is

$$
C_{i}=\frac{2\left|\left\{j, k \in N_{i} ; e_{j k} \in E\right\}\right|}{k_{i}\left(k_{i}-1\right)} .
$$

Here, $C_{i}$ is the clustering coefficient of node $i$, node $j$ and node $k$ are the neighboring nodes of node $i, e_{j k}$ is the edge in the undirected graph $G$, and $k_{i}\left(k_{i}-1\right)$ is the number of possible connections in the $N_{i}$.

(3) Measuring PageRank. PageRank algorithm, also known as network ranking algorithm, is used to measure the importance of web pages [20, 21]. Yu and Lu used PageRank algorithm to reveal the basic vocabulary within the discipline [22]. $\mathrm{Gu}$ and $\mathrm{Xu}$ proposed LTWPR (located and TFweighted PageRank) algorithm based on PageRank algorithm, which can extract keywords of text more accurately [20]. The SQT-PageRank core patent discovery method proposed by Xuis is superior to the PageRank algorithm [23]. In this study, paper keywords are used to calculate ranking, that is, the text topic ranking algorithm TopicRank (TR) [24]. TR is defined as follows:

$$
\operatorname{TR}\left(k_{i}\right)=\frac{1-d}{N}+d * \sum_{k_{j} \in M\left(k_{i}\right)}^{n} \frac{\operatorname{TR}\left(k_{j}\right) * \operatorname{Weight}\left(k_{j}\right)}{\operatorname{Degree}\left(k_{j}\right)},
$$

where $\operatorname{TR}\left(k_{i}\right)$ is the TopicRank value of keyword $i$, $\mathrm{d}$ is the damping factor, which is generally 0.85 by default, $N$ is the total number of keywords, $M\left(k_{i}\right)$ is the set of keywords connected with keyword $i$, Degree $\left(k_{j}\right)$ is the degree of keyword $j$, and Weight $\left(k_{j}\right)$ is the weight of edge $\left(k_{i}, k_{j}\right)$.

(4) Structure Hole and Constraint. Burt proposed the structure hole theory, which means that one or some individuals in a social network are directly connected to some individuals but not to others, i.e., there is no direct relationship or the relationship is intermittent, and the network as a whole appears as if there is a hole in the network structure [25].

In structural hole theory, the "limit degree" of an individual is the ability of that individual to use the structural hole in his or her own network. The larger the value is, the stronger the node's constraint is. Thus, nodes have access to fewer sources of information, which is not conducive to generating structural advantages. For a node's structural hole, it is obtained using 1-constraint. For node $i$ and its neighborhood $N_{\mathrm{i}}$, the constraint of $i$ is calculated as follows [25]:

$$
\text { Constraint }_{i}=\sum_{j \in N_{i}}\left(p_{i j}+\sum_{q} p_{i q} p_{q j}\right)^{2}, \quad q \neq i, j,
$$

where $j$ is the neighboring nodes of node $i, \mathrm{q}$ is the nodes other than node $i$ and $j, p_{i j}$ represents the proportion of direct links between nodes $i$ and $j$ to the total links of node $i$, and $p_{i q} p_{q j}$ refers to the proportion of links of node $i$ indirectly connected to node $j$ through node $q$ to the total links of node $i$.

2.2.3. Measuring Scientific Performance. There are two common ways to measure scientific performance: productivity and impact $[18,26,27]$. We use the average citation count to measure the impact of knowledge network elements, and the number of articles of knowledge network elements is chosen as the productivity measure.

\section{Data Statistics}

Figure 1 shows the trend of the number of papers issued by the Q1 area journals of sports science. From 2000 to 2020, the number of papers issued by the Q1 area journals of sports science in China was 45472 . From the figure, we can find that the annual numbers of paper showed a trend of rising and then falling. Before 2007, it had an upward trend. In 2007, a total of 2998 papers were published. Then, the number of annual papers decline after 2007. A study was conducted using the volume of articles published in 567 journals (approximately 1.05 million papers) across 25 disciplines included in the CSSCI during the period 2010-2019. It finds that the number of C-journal papers in all disciplines declined from 2010 to 2019. The decline in sports science, political science, economics, library, intelligence and literature, etc., is obvious. The number of papers published in sport science journals decreased by $43 \%$, which was the largest decrease among all subjects. The decline in the number of articles published in C-journal may be due to a 


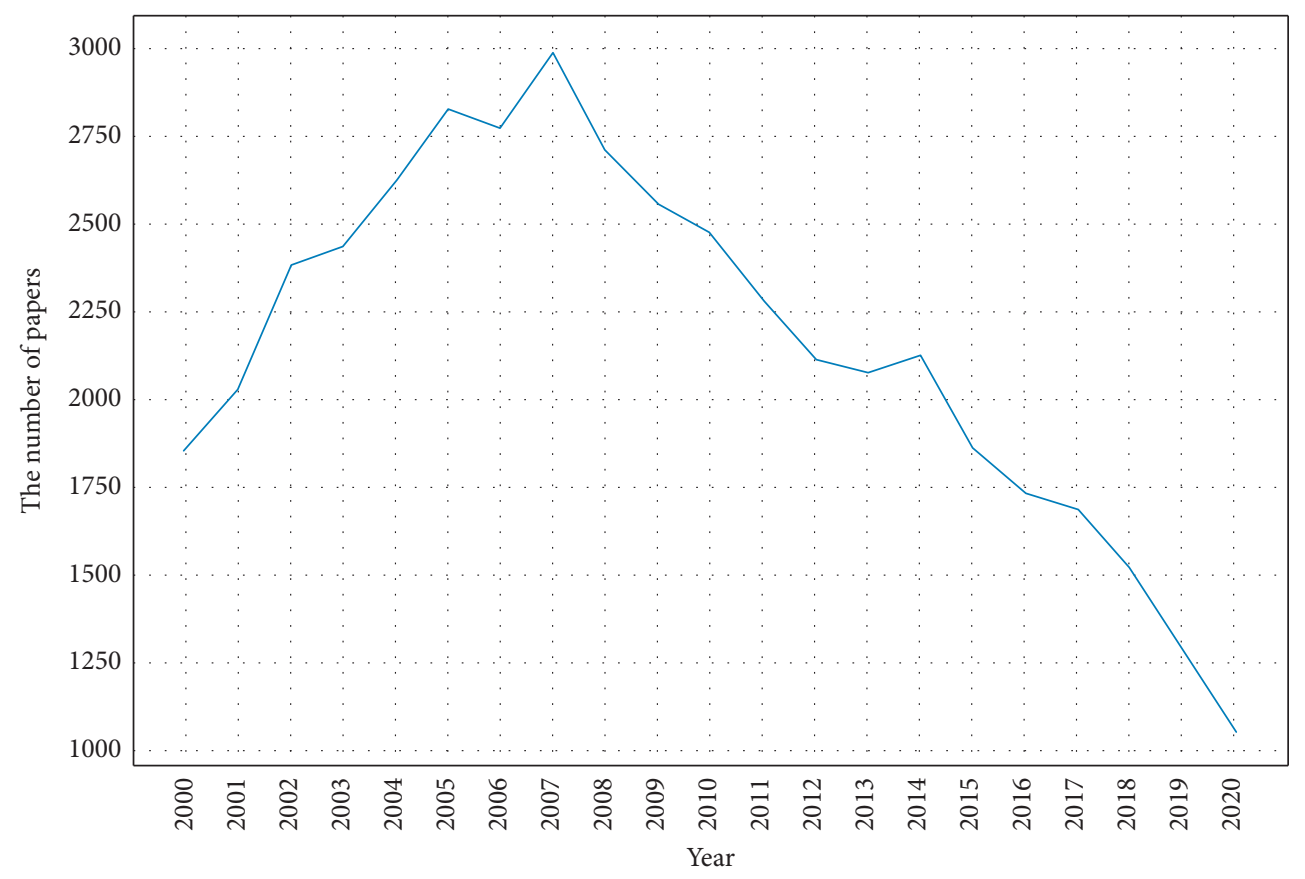

Figure 1: The trend of papers (2000-2020).

shift in the journal's strategy from pursuing high production to high quality.

\section{Analysis of Research Hotspots}

With the development of social network research, social network visualization software has gradually increased, such as UCINET, CiteSpace, Pajek, and VOSviewer. The advantage of VOSviewer is the "cooccurrence clustering," i.e., the simultaneous occurrence of two things indicates their relatedness to a certain extent. The more the number of simultaneous occurrence is, the greater the relatedness is [28]. Run the VOSviewer software for cooccurrence of the processed data, as shown in Figure 2. From 2000 to 2020, the keywords with frequency greater than 150 were screened, and the keyword pairs with cooccurrence frequency greater than 3 were constructed to cooccurrence network.

Table 1 is the calculation of the dividing line formula of high- and low-frequency keywords based on Donohue, and the table of high-frequency keywords whose keyword frequency exceeds the minimum threshold of 268. In the keyword frequency statistics, we removed the keywords with low specific reference (such as China, sports, research, analysis, influence, etc.) and merged the synonyms (such as 2008 Olympic Games, 29th Olympic Games, and Beijing Olympic Games; colleges and universities; and the Olympic Movement and the Olympics)

4.1. Microperspective: Hotspots Analysis. Table 1 shows that the research hot topics of sports science are competitive sports, school sports, sports management, physical education, mass sports, sports culture, national traditional sports, sports industry, sports history, sports economy, sports teaching, Olympic movement and Olympic Games, national fitness, rat, college sports, animals experiment, etc. The high-frequency keywords of sports management, physical education, national traditional sports, sports history, and sports economy have obvious disciplinary nature, while competitive sports, school sports, mass sports, sports culture, sports industry, physical education, and animal experiments are all classical directions of sports science research in China. The research hot groups are college students, athletes, teenagers, and physical education teachers. The research hot programs include traditional national sports of martial arts, competitive sports of football, and table tennis.

Competitive sports is the keyword with the highest frequency in the recent 20 years. Since the development of competitive sports in China, competitive sports has always been the important research direction of sports in China and has become an absolute hotspot in the field of sports research in China. As shown in Figure 3, the competitive sports showed a trend of rising and then falling, with a rapid increase around 2008. By analyzing the papers about competitive sports in the past 20 years, the research on competitive sports has become an absolute upsurge in this Olympic cycle since the Beijing Olympic Games was held in China. As time goes by, the popularity of competitive sports has declined. The research about competitive sports focuses on the high-quality development of competitive sports in the new era [29], technology-led competitive sports [30], and the Winter Olympics [31, 32], and it is not limited to the study of the development status and path of competitive sports in the perspective of the Olympic Games.

Figure 4 shows the trend of keyword, mass sports. Mass sports is also one of the research hotspots in the Q1 area of sports science in China from 2000 to 2020. Before 2008, there was less research on mass sports, and after 2008, it 


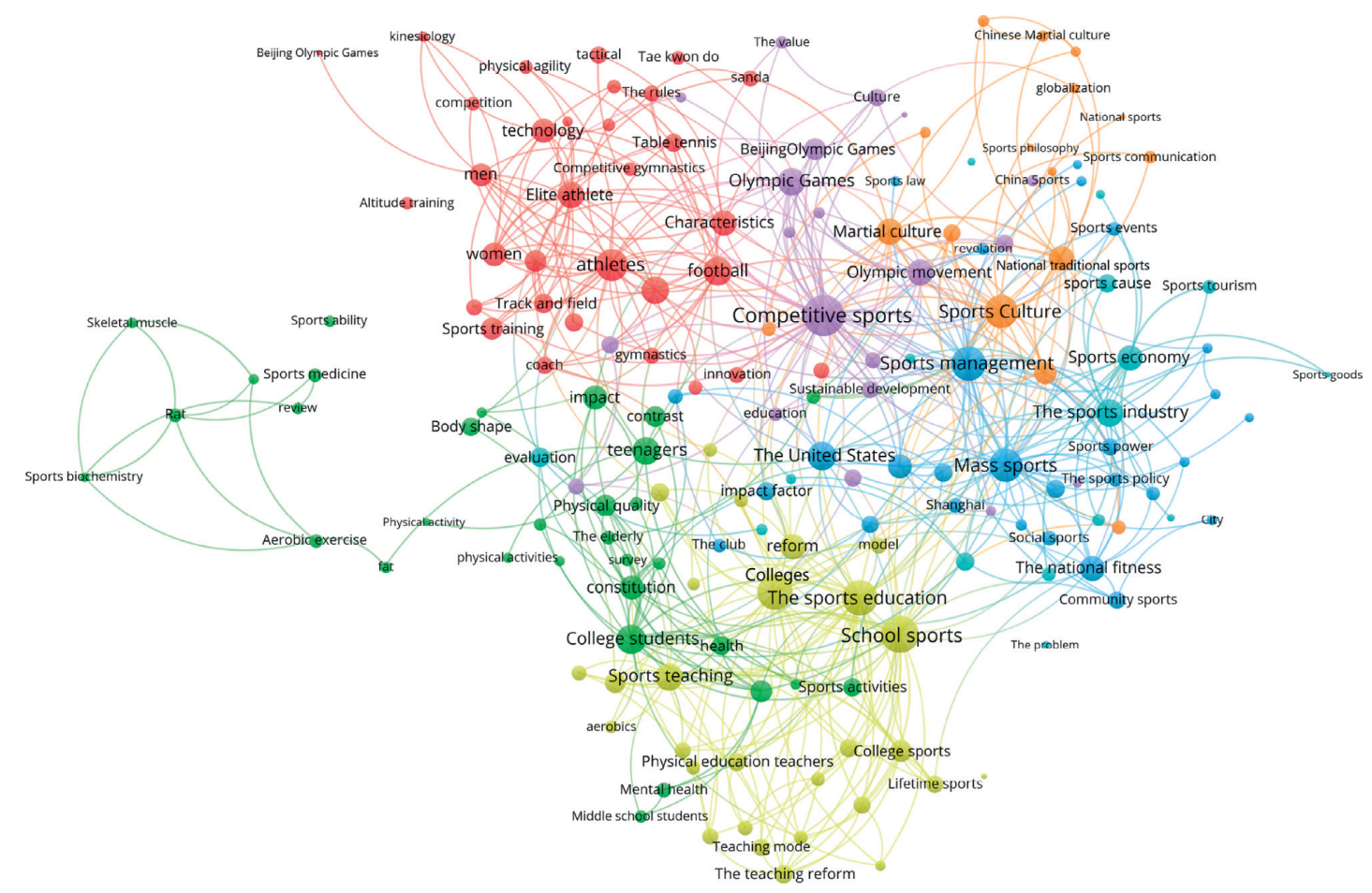

FIgURE 2: Keyword cooccurrence network.

TABle 1: High-frequency keywords.

\begin{tabular}{lcc}
\hline No. & Keyword & Frequency \\
\hline 1 & Competitive sports & 1853 \\
2 & School sports & 1316 \\
3 & Sports management & 1262 \\
4 & Physical education & 1243 \\
5 & Mass sports & 1240 \\
6 & Sports culture & 1104 \\
7 & Traditional national sports & 993 \\
8 & Colleges & 962 \\
9 & Sports industry & 924 \\
10 & College students & 839 \\
11 & Athletes & 808 \\
12 & Martial arts & 797 \\
13 & Sports history & 797 \\
14 & Sports economy & 778 \\
15 & Sports teaching & 741 \\
16 & Olympic movement & 590 \\
17 & The Olympic games & 589 \\
18 & Football & 585 \\
19 & National fitness & 511 \\
20 & Beijing Olympic games & 496 \\
21 & The United States & 471 \\
22 & Rat & 459 \\
23 & Teenagers & 446 \\
24 & Basketball & 446 \\
25 & Excellent athletes & 411 \\
26 & Sports training & 387 \\
27 & Physical education curriculum & 373 \\
28 & PE teachers & 362 \\
29 & Women & 343 \\
30 & Physical exercise & 329 \\
\hline & &
\end{tabular}

TABle 1: Continued.

\begin{tabular}{lcc}
\hline No. & Keyword & Frequency \\
\hline 31 & Constitution & 319 \\
32 & Sports sociology & 299 \\
33 & Japan & 297 \\
34 & Men & 287 \\
35 & Culture & 286 \\
36 & College sports & 283 \\
37 & Animal experiments & 280 \\
38 & Table Tennis & 277 \\
\hline
\end{tabular}

entered an upward phase, reaching its peak in 2014-2015. In recent years, many Chinese scholars have focused their research on mass sports, such as national fitness [33] and leisure sports [34]. The research in this field is in line with the characteristics of this era. However, it is different from competitive sports. The research focus of mass sports is still in its initial stage, with the majority of qualitative research, such as countermeasures research $[33,35]$ and development research [34].

Figure 5 is the trend of keyword, sports management. Sports management is one of the disciplines of sports science. It applies management theories and methods to study the coordination of sports organizations in order to achieve the predetermined goals of sports [36], thus came into being related research on sports management. To be specific, sports management is an activity process in which managers in sports organizations coordinate the activities of others and play the role of various resources to achieve predetermined goals through the implementation of planning, 


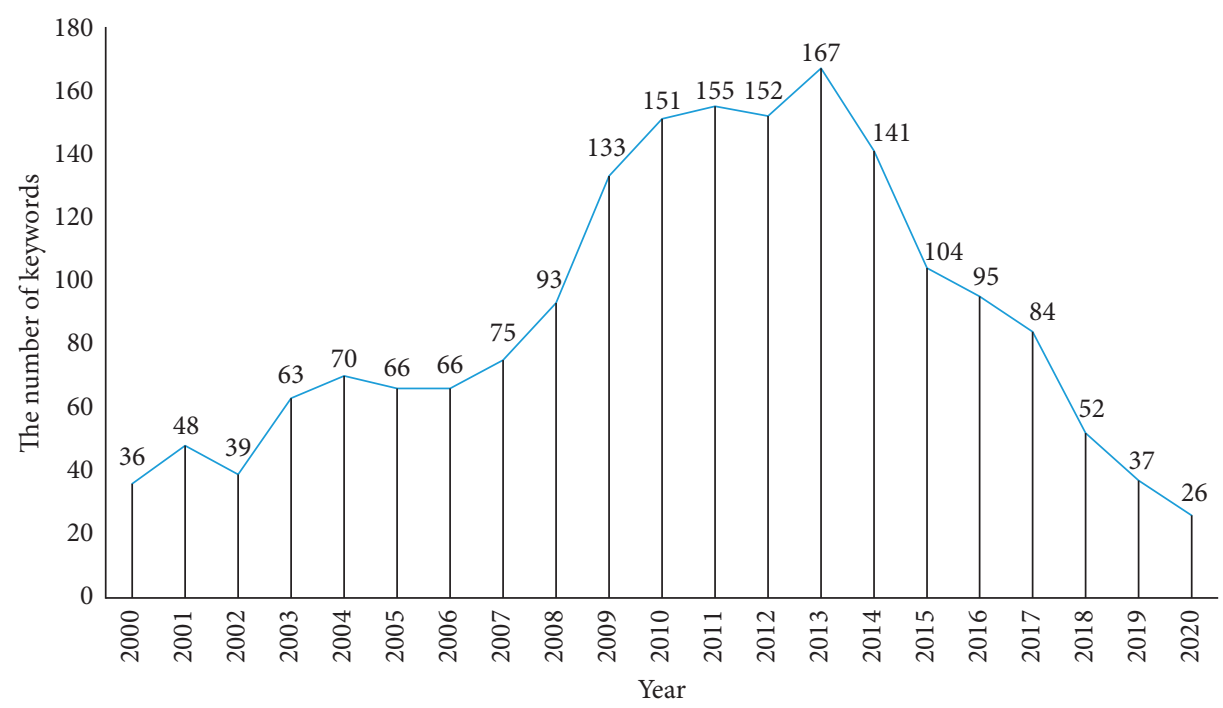

FIGURE 3: The trend of keyword, competitive sports.

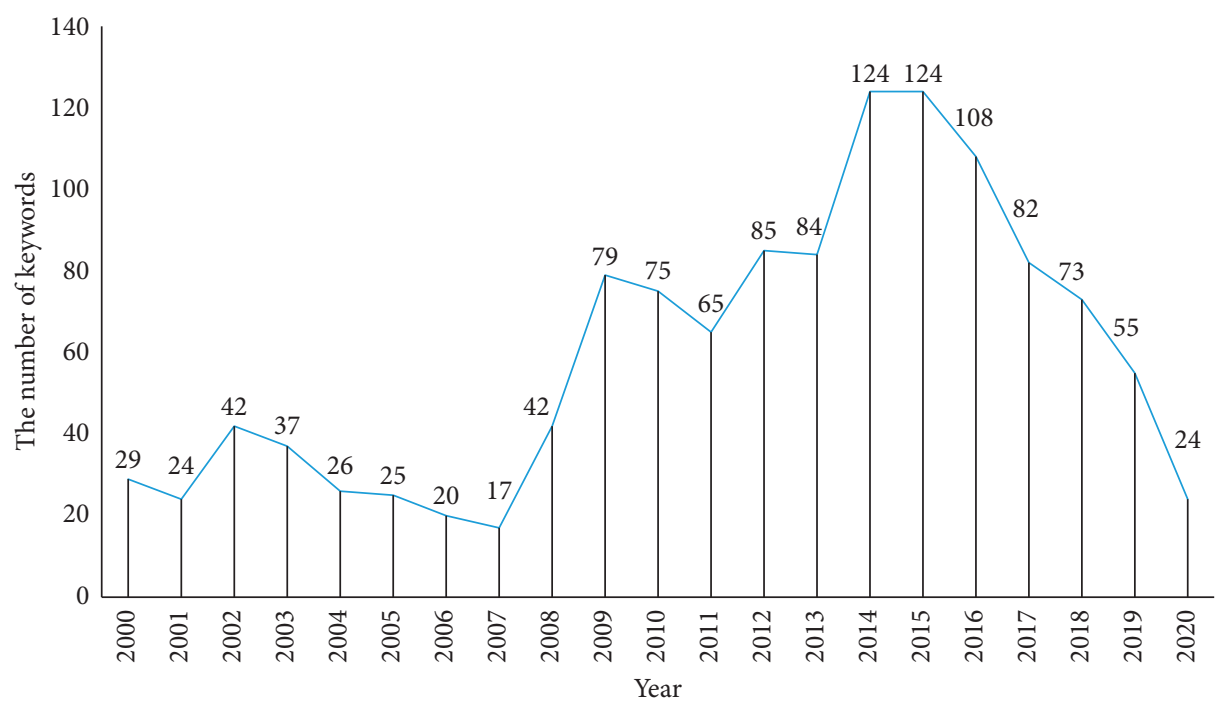

FIGURE 4: The trend of keyword, mass sports.

organization, leadership, and control functions for the object of sports management [36]. In sports management, mass sports management, competitive sports management, and school sports management are the main research contents [37]. The change trend of sports management is same as that of mass sports and competitive sports. After 2008, the number of its related studies increased rapidly and then declined after 2013-2014. Compared with school sports, there are more researches on mass sports and competitive sports in sports management. The research on competitive sports in sports management focuses on the research on management system $[38,39]$ and the research on development [40, 41], which deeply analyzes the institutional problems of competitive sports, optimizes the development path of competitive sports, and promotes the vigorous development of competitive sports. Research on mass sports in sports management focuses on sports policies [42, 43], community sports [44], and the elderly sports and the disabled sports $[45,46]$. From the perspective of management, it analyzes how China develops mass sports and how to give correct policy guidance to mass sports.

Figure 6 shows the trend of keyword, school sports and physical education. As a keyword of high frequency, school sports has always been one of the research key points in the field of sports science. As an important branch of sports science, school sports and its derivative keywords account for almost half of the high-frequency keywords. As shown in Figure 6, the emergence of school sports is bound to accompany the emergence of physical education, and they complement each other and develop together. In the past 20 years, it has been found that the research on school sports has a fluctuation. Many scholars' researches on school sports have mainly focused on teaching reform [47-49]. With the continuous development of sports teaching reform research, 


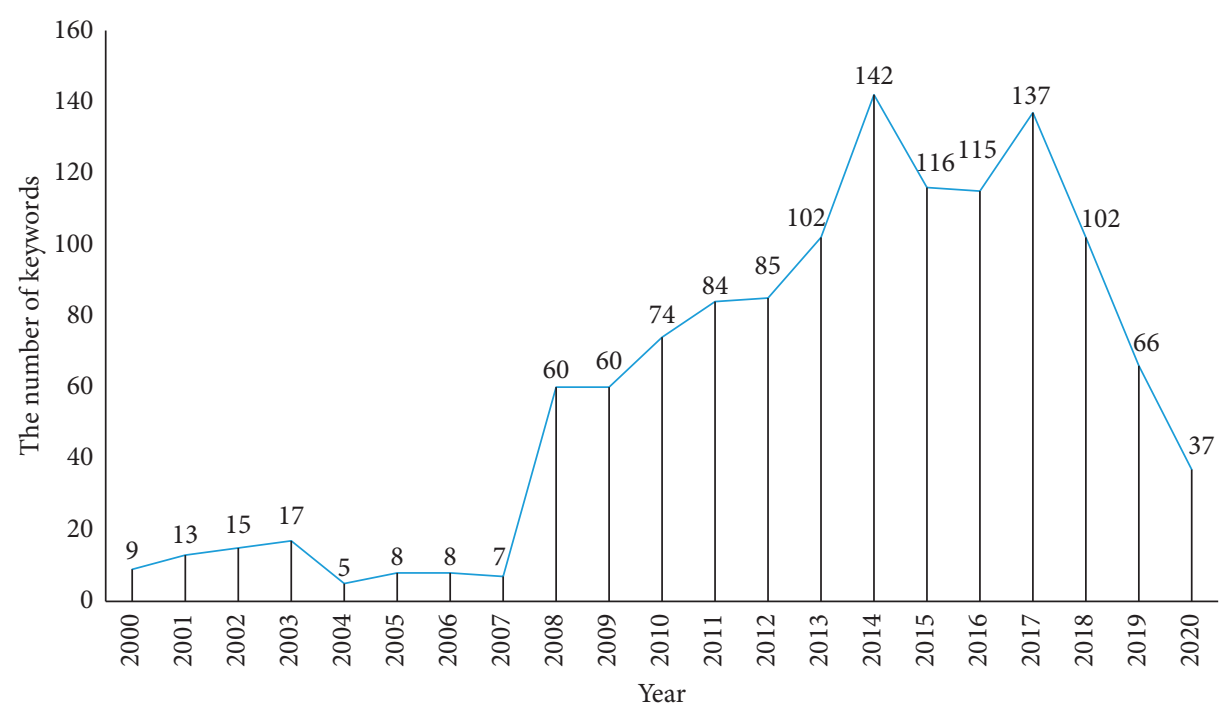

FIGURE 5: The trend of keyword, sports management.

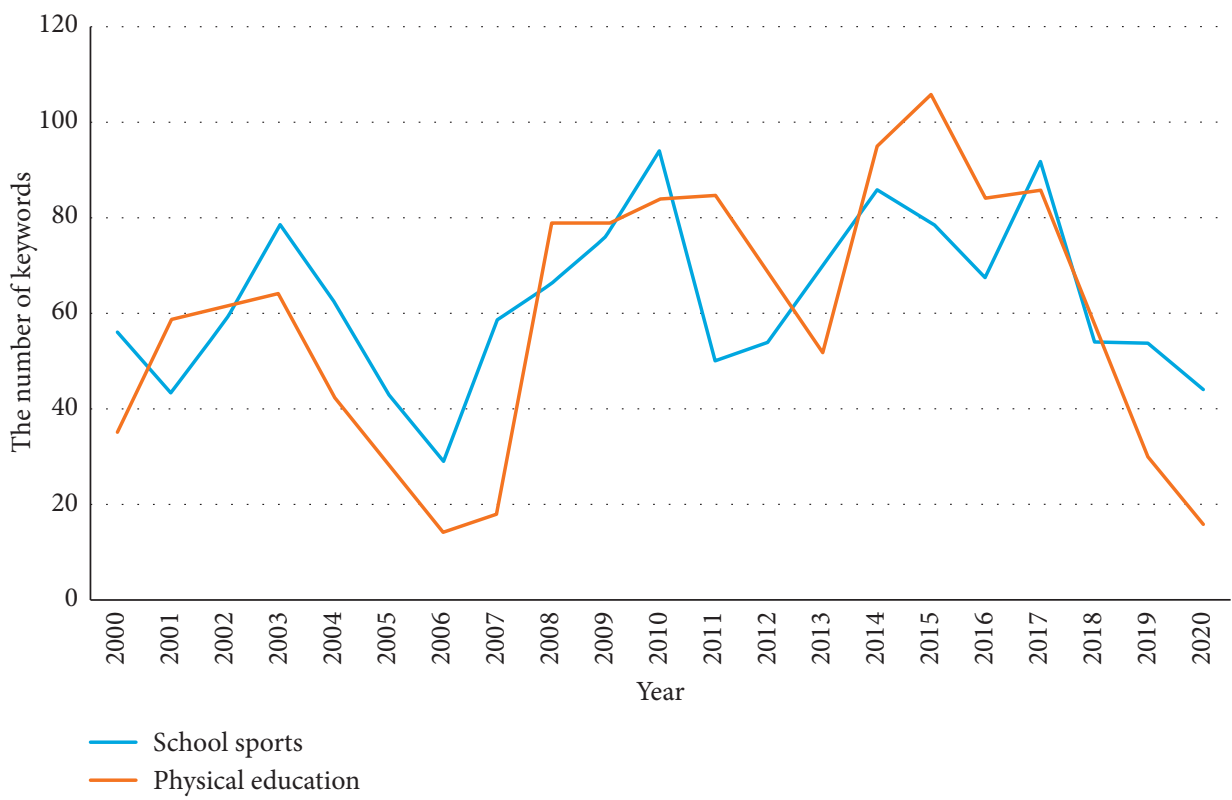

Figure 6: The trend of keyword, school sports and physical education.

almost all aspects of school sports are covered from teaching idea to teaching pattern.

4.2. Macroperspective: Cluster Analysis. According to the clustering results of VOSviewer in Figure 2, it can be seen that the research hotspots of sports journals in Q1 area of China from 2000 to 2020 mainly focus on six categories: red clustering belongs to the category of sports training; purple clustering belongs to the category of competitive sports; orange clustering belongs to the category of national traditional sports; sports medicine and sports biochemistry of green clustering belong to the category of sports human science; blue clustering belongs to the category of mass sports and sports management; cyan clustering belongs to the category of sports industry and sports economy; yellow clustering belongs to the category of school sports and physical education.

(1) Sports training of red clustering and physical education of yellow clustering belong to physical education and training. Physical education and training is an interdisciplinary and comprehensive discipline composed of traditional physical teaching theories and methods and sports training, which mainly research the system of basic theories and methods of physical education and sports training [50]. As a subdiscipline of physical education and training, sports training is an important part of competitive sports. Athletes are trained scientifically under the 
guidance of coaches to improve their competitive level and sports results, so as to show their ability and spirit in the competition and win glory for the country. Physical education achieves the goal of education by promoting people's physical and mental development through sports; its research focuses on school sports and college physical education.

(2) The orange clustering belongs to national traditional sports. Traditional sports is a kind of fitness and entertainment activity with strong ethnic cultural color. It mainly focuses on physical movement and the understanding of the human body of various nationalities and also a special education method used by people to seek to enhance physical skills training [51]. According to the clustering results of VOSviewer, the research topics of national traditional sports are mainly composed of sports culture, traditional sports, and the Olympic Games. The inheritance and internationalization of traditional national sports have always been the focus of traditional sports research in China.

Fan and $\mathrm{Yu}$ studied the inheritance of traditional martial arts and analyzed its current situation, problems, and countermeasures [52]. Li and Guo studied the inheritance and international dissemination of martial arts. They pointed out that martial arts culture should give full play to its advantages and be the pioneer in the process of international dissemination of Chinese martial arts and explore the frontier position of the international promotion of Chinese martial arts [53]. In addition to the research on the inheritance and internationalization of martial arts, the Chinese sports science has a lot of researches on the Olympic-entering of the traditional national sport, martial arts. Its essence of those researches is still the internationalization and inheritance of martial arts. Liu et al. deeply analyzed the difficulty of Olympic-entering of martial arts because of the low popularity of martial arts and the esoteric martial arts culture [54]. Hong et al. pointed out that the cultural differences between China and Western countries and the degree of international development made martial arts difficult to become an official event in the Olympic Games [55]. As one of the traditional sports programs, we should take the cultural connotation as the core, promote its traditional spirit, popularize the education of martial arts, and move towards the forefront of the internationalization of national traditional sports.

(3) Green clustering belongs to sports human science. Sports medicine and sports biochemistry are subordinate to the secondary disciplines of sports human science. Sports human science is a subject which applies the theory and method of anatomy, physiology, nutrition, and biochemistry to study the influence of sports on human body shape, structure, and physiological function, as well as the law and measure of health care in sports. As one of the secondary disciplines of sports science, human sports science has fewer hot topics compared with physical education and training, sports humanities and sociology, and national traditional sports from the perspective of knowledge map of keywords cooccurrence.

(4) The purple, blue, and cyan clustering all belong to sports humanities and sociology. Sports humanities and sociology is a comprehensive discipline developed on the basis of the humanities and social sciences, which studies the essential issues of sports, such as the relationship between sports and human beings, sports and society, and the basic laws, through the theories and methods of the humanities and social sciences [56]. The main research directions of sports humanities and sociology include competitive sports, school sports, social sports (national fitness problem), sports industry, and market, and sports management is also one of the research directions of sports humanities and sociology [57].

Competitive sports are a process of sports activities that maximizes the potential of athletes in physical, psychological, and intellectual aspects; while developing the body comprehensively, the main purpose is to climb the peak of sports technique and create excellent sports performance [58]. Competitive sports is an important way to highlight the national sports strength. Athletes as the main body of competitive sports, its cultivation and training have always been a hot topic $[59,60]$.

School sports is a discipline that combines sports with education and cultivate teachers to follow the national policy of all-round development of moral, intellectual, physical, social, and aesthetic education. Based on the characteristics of the students' physical and mental development, by means of proper physical exercises and health knowledge, through the physical education curriculum, physical exercise, sports competition, and so on, school sports is a planned and organized education activity, which dedicates to strengthen students' physique and cultivate students' consciousness, interest, habit and ability of lifetime sports, and help them become a socialist builders and defenders with comprehensive development in moral, intelligence, sports, aesthetics, and labour education [61]. Related concepts of physical education run through the work of school sports; the research topics are also centered on the two large pieces of education and teaching. Education pays attention to process, teaching focuses on results, and research focuses on teaching mode, effect, and reform, promoting the development of school sports to achieve the aim of students' all-round development. For example, Zhou et al. pointed out that the close connection between teaching materials and society and life should be strengthened in their research on the reform of physical education content in universities, and that there should be not only competitive programs but also fitness, entertainment, and traditional 
Chinese national sports, so as to realize a good transition from school sports to social sports [62].

Sports Management and Mass Sports. Sports management is a discipline that applies management theories and methods to study the coordination of sports organizations to achieve predetermined sports goals [37]. Mass sports refer to sports activities with a wide range of contents and various forms that are voluntarily participated by ordinary people for the purpose of physical improvement, physical fitness, entertainment, leisure, and social interaction and generally do not seek to achieve high level of athletic performance [63]. Research on mass sports in sports management focuses on analyzing the realistic conditions and restrictive factors of mass sports in China from the perspective of management, so as to optimize the development path of mass sports in China [42-46].

Sports Industry and Economy. Sports industry refers to the collection of the same kind of economic activities and the synthesis of the same kind of economic sectors that provide sports products for the society. Although China's sports industry started late, it has developed rapidly. The field and scale of the industry have been expanded; the quality benefits have also been improved significantly. On the basis of the sound modernization system of sports industry, the highquality development of sports industry has become the focus of research. Li and Liu analyzed the development concept, topics context, and logical thinking of the sports industry [64]. Guo and Ren analyzed the inner logic and basic connotation of the high-quality development in the new era and pointed out the path selection of high-quality development of sports industry: promoting the supply-side structural reform in sports industry, strengthening sports market entity, perfecting construction of sports market system, promoting the efficient integration of sports industry and other industries and the spatial distribution optimization, and improving the system of development and policy in sports industry [65].

To sum up, the research hotspots of Q1 area journals in the past 20 years involve physical education and training, sports humanities and sociology, sports human science, and national traditional sports.

\section{Analysis of the Relationship between Knowledge Networks and Scientific Performance Correlations}

5.1. Descriptive Statistics of Variables. Table 2 is a descriptive statistical table of variables. For the sample, the mean value of the number of articles is 28.6, standard deviation 85.7, minimum value 6 , ad maximum value 2407 . The mean value of citation counts is 21.7 . The mean values of sample degree centrality, betweenness centrality, and closeness centrality are 42.7, 2994.6, and 0.4, respectively. The mean value of PageRank for the sample is only 0.00024 , with a standard deviation of 0.00045 . The means of clustering coefficient and structural holes are 0.23 and 0.945 , respectively.
TABLE 2: Descriptive statistics of variables.

\begin{tabular}{lcccc}
\hline Variable & Mean & Std. dev. & Min & Max \\
\hline Paper counts & 28.64119 & 85.66474 & 6 & 2405 \\
Average citation & 21.68361 & 11.71496 & 2.5 & 148.25 \\
$\begin{array}{l}\text { Degree centrality } \\
\text { Betweenness }\end{array}$ & 42.72149 & 68.7952 & 3 & 1537 \\
centrality & 2994.606 & 22082.97 & 0.723307 & 1012664 \\
Closeness & & & & \\
centrality & 0.4125856 & 0.038428 & 0.286623 & 0.3840 \\
PageRanks & 0.0002411 & 0.000454 & 0.000049 & 0.013906 \\
Clustering & 0.2286381 & 0.0823648 & 0 & 0.7 \\
Structural holes & 0.9457353 & 0.0335163 & 0.6375047 & 0.9974905 \\
\hline
\end{tabular}

${ }^{*}$ Obs $=4147$.

5.2. Correlation Analysis of Independent Variables. Table 3 shows the correlation test results of the independent variables and the correlation coefficients between closeness centrality and degree centrality; betweenness centrality and degree centrality are high; closeness centrality and betweenness centrality Pearson correlation coefficient is low, only 0.3321 . Leydesdorff measured degree centrality and betweenness centrality in a network with sample size of 7379 and degree centrality and closeness centrality, and closeness centrality and betweenness centrality correlation coefficients were $0.509,0.651$, and 0.210 , respectively. From the table, we can also find that the correlation coefficients between PageRank and three centralities are high, which is an expected result. The PageRank uses the degree of the neighboring nodes of the direct node to calculate and thus the two show high correlation. From the variance inflation factor (VIF) test, we can find that there is a serious problem of multicollinearity in the model, so we removed the variables degree centrality and PageRanks. After removing the variables and conducting the VIF test again, the VIF of all variables is less than 5 .

5.3. Associations between Knowledge Network and Research Influence. Table 4 shows the multiple regression analysis of knowledge network features and performance. From the table, we can learn that only the correlation of betweenness centrality and average citation counts is not significant, and the rest of the results are significant at different levels. Although the correlation of betweenness centrality and paper counts is significant, the correlation coefficient was only 0.0032. In the multiple regression of knowledge network features and impact, we can find that when a node is in the core position of the network, i.e., when the closeness centrality goes higher, its impact will increase. Similarly, when the nodes of a knowledge network are clustered into groups or in the structural hole position, they are conducive to the higher citations and increased impact. In the multiple regression of knowledge network features and productivity, closeness centrality also shows a significant positive correlation with productivity. Interestingly, clustering coefficients and structural holes present different results from those in the impact analysis. When knowledge network nodes cluster into groups with other nodes, they instead reduce their productivity, and when nodes are in structure holes, their 
TABLE 3: Independent variable correlation test and VIF.

\begin{tabular}{lcccccr}
\hline & & 1 & 2 & 3 & 4 & 5 \\
\hline 1 & Degree centrality & - & & & & \\
2 & Betweenness centrality & 0.8139 & - & & 39.75 \\
3 & Closeness centrality & 0.6509 & 0.3321 & - & 8.41 \\
4 & PageRanks & 0.9723 & 0.8996 & 0.5446 & - & 3.86 \\
5 & Clustering & -0.5139 & -0.2454 & -0.4942 & -0.4341 & 54.20 \\
6 & Structural holes & 0.4862 & 0.1788 & 0.7743 & 0.3840 & -0.6369 \\
\hline
\end{tabular}

TABLE 4: Multiple regression analysis.

\begin{tabular}{|c|c|c|c|c|}
\hline \multirow{2}{*}{ Knowledge network measure $(\mathrm{Obs}=4147)$} & \multicolumn{2}{|c|}{ Average citation } & \multicolumn{2}{|c|}{ Paper counts } \\
\hline & $\beta$ & Std. err. & $\beta$ & Std. err. \\
\hline Betweenness centrality & -0.0000146 & $8.69 e-06$ & $0.0031839^{* *}$ & 0.0000254 \\
\hline Closeness centrality & $65.16445^{* *}$ & 7.646053 & $394.5624^{* *}$ & 22.35862 \\
\hline Clustering & $10.01557^{* *}$ & 2.826052 & $-129.9039^{* *}$ & 8.263953 \\
\hline Structural holes & $29.53742 *$ & 9.575936 & $-151.2^{* *}$ & 28.00199 \\
\hline
\end{tabular}

${ }^{*}$ Correlation is significant at the 0.05 level (2-tailed). ${ }^{* *}$ Correlation is significant at the 0.01 level (2-tailed).

productivity decreases. This may be due to the fact that the nodes in the structure hole position are only associated with a small number of nodes and are not hotspots.

\section{Discussion and Conclusion}

The research in this paper is helpful to understand the research status quo and development situation of the field and provide decision-making and reference information for the selection of thesis topics, research projects, and discipline planning in sports science. Through keywords frequency analysis and social network analysis method, we can draw the following conclusions:

First, through word frequency method, we find 38 research hotspots of Q1 area journals of sports science in China, such as competitive sports, school sports, sports management, sports education, and mass sports. There are four research focus groups: college students, athletes, teenagers, and physical education teachers, and we found three hot research programs: martial arts in traditional national sports and football and table tennis in competitive sports. Therefore, it is necessary to understand the current situation of sports science and maintain the development of physical education and training, sports humanities, and sociology and national traditional sports. At the same time, we need to accelerate the development of sports human science, which is the focus of future research.

Secondly, the characteristics of knowledge network elements were significantly associated with scientific research performance, except for betweenness centrality, which was not significantly related to average citation.

\section{Data Availability}

The original data used in this paper are from the Chinese Social Sciences Citation Index Database.

\section{Conflicts of Interest}

The authors declare that they have no conflicts of interest.

\section{Authors' Contributions}

Linxiao Ma was responsible for writing the original draft, review and editing, and literature collection. Yuzhu Wang was responsible for determining the framework of paper, funding acquisition, and review and editing. Yue Wang was responsible for writing the original draft. Ning Li was responsible for review and editing. Sai-fu Fung was responsible for funding acquisition. Lu Zhang and Qian Zheng did calculations. All authors discussed and interpreted results.

\section{Acknowledgments}

This work was supported in part by the National Key R\&D Plan (2020YFC2007003).

\section{References}

[1] D. Lazer, A. Pentland, L. Adamic et al., "Social science: computational social science," Science, vol. 323, no. 5915, pp. 721-723, 2009.

[2] Y. Chen, W. Tian, and J. Wu, "Research on the visual analysis method of topic domain research hotspots tracking and trend forecasting," Information Theory and Practice, vol. 40, no. 6, pp. 117-121, 2017.

[3] F. Luo and B. Lu, "Dynamic analysis of China's circular economy research from the perspective of knowledge mapping," Journal of Statistics and Information, vol. 32, no. 3, pp. 109-113, 2017.

[4] J. Chu and Q. Qian, "Research on knowledge management based on word frequency analysis in recent 10 years," Information Science, vol. 32, no. 10, pp. 156-160, 2014.

[5] Y. Li and C. Jiang, "Hotspots and future trends of physical education research in China in recent five years," Journal of Wuhan Institute of Physical Education, vol. 53, no. 4, pp. 19-25, 2019. 
[6] L. Jiang, Z. Wang, and X. Wang, "Key words analysis of sports humanistic sociology papers based on CSSCI," Journal of Southwest University for Nationalities (Humanities and Social Sciences Edition), vol. 35, no. 1, pp. 229-238, 2014.

[7] J. C. Donohue, Understanding Scientific Literatures: A Bibliometric Approach, Massachusetts Institute of Technology Press, Cambridge, MA, 1972.

[8] C. Lyndon, Freeman. The History of Social Network Analysis, China People's University Press, Beijing, China, 2008.

[9] F. Xiong, X. Wang, S. Pan, H. Yang, H. Wang, and C. Zhang, "Social recommendation with evolutionary opinion dynamics," IEEE Transactions on Systems, Man, and Cybernetics: Systems, vol. 50, no. 10, pp. 3804-3816, 2020.

[10] Y. Hu, F. Xiong, S. Pan, X. Xiong, L. Wang, and H. Chen, "Bayesian personalized ranking based on multiple-layer neighborhoods," Information Sciences, vol. 542, pp. 156-176, 2021.

[11] A. Abbasi, J. Altmann, and L. Hossain, "Identifying the effects of co-authorship networks on the performance of scholars: a correlation and regression analysis of performance measures and social network analysis measures," Journal of Informetrics, vol. 5, no. 4, pp. 594-607, 2011.

[12] E. Y. Li, C. H. Liao, and H. R. Yen, "Co-authorship networks and research impact: a social capital perspective," Research Policy, vol. 42, no. 9, pp. 1515-1530, 2013.

[13] E. Yan and D. Ying, Applying Centrality Measures to Impact Analysis: A Coauthorship Network Analysis, John Wiley \& Sons, Inc., Hoboken, NJ, USA, 2009.

[14] J. Guan, Y. Yan, and J. J. Zhang, "The impact of collaboration and knowledge networks on citations," Journal of Informetrics, vol. 11, no. 2, pp. 407-422, 2017.

[15] L. Egghe, "Theory and practise of the g-index," Scientometrics, vol. 69, no. 1, pp. 131-152, 2006.

[16] A. Abbasi, R. T. Wigand, and L. Hossain, "Measuring social capital through network analysis and its influence on individual performance," Library \& Information Science Research, vol. 36, no. 1, pp. 66-73, 2014.

[17] F. Xiong, W. Shen, H. Chen, S. Pan, X. Wang, and Z. Yan, "Exploiting implicit influence from information propagation for social recommendation," IEEE Transactions on Cybernetics, vol. 50, no. 10, pp. 4186-4199, 2020.

[18] S. Guoan, J. Luo, J. Sun, F. Rong, and C. Zhang, "Analysis of the academic impact of articles in journal of Xi'an Jiaotong university (social science edition)," Journal of Statistics and Information, vol. 24, no. 07, pp. 92-96, 2009.

[19] D. J. Watts and S. H. Strogatz, "Collective dynamics of 'smallworld' networks," Nature, vol. 393, pp. 440-442, 1998.

[20] Y. Gu and M. Xu, "News keyword extraction algorithm based on PageRank," Journal of University of Electronic Science and Technology of China, vol. 46, no. 5, pp. 777-783, 2017.

[21] X. Guo and X. Zhang, "Hot topics and future prospects of education research on "one belt and one road"-based on CSSCI literature analysis (2013-2019)," Journal of Xi'an University of Finance and Economics, vol. 33, no. 4, pp. 98104, 2020.

[22] F. Yu and W. Lu, "Key words co-occurrence network perspective of discipline basic vocabulary discovery," Library and Information Service, vol. 63, no. 9, pp. 95-100, 2019.

[23] C. Xu, Research on Core Patent Discovery Based on Improved PageRank Algorithm, Shanxi University, Taiyuan, China, 2020.

[24] H. Zhao, "Centrality and power embodiments: a study on the generation path of network media power based on social network analysis," Journalism and Communication Research, vol. 20, no. 03, pp. 50-63+127, 2013.

[25] R. S. Burt, Structural Holes, Harvard University Press, Cambridge, MA, USA, 1992.

[26] J. Guan and L. Pang, "Bidirectional relationship between network position and knowledge creation in Scientometrics," Scientometrics, vol. 115, no. 1, pp. 201-222, 2018.

[27] C. Damien, D. Arnaud, L. Catherine et al., "The impact of a researcher's structural position on scientific performance: an empirical analysis," PLoS One, vol. 11, no. 8, Article ID e0161281, 2016.

[28] L. Zuo and X. Xiao, "Comparison of knowledge graph visualization tools VosViewer and NWB tool," Information Science, vol. 33, no. 2, pp. 95-99, 2015.

[29] G. Yang, "On the new development of Chinese competitive sports in the new era," Sports Culture Guide, vol. 3, pp. 11-16, 2019.

[30] W. Tian and F. Lianshi, "Research on the relationship between sports science and technology innovation and competitive sports training level," Journal of Beijing Sport University, vol. 6, pp. 825-827, 2003.

[31] X. Yao, "Experience and inspiration of ice and snow resources development in host cities of the Winter Olympic Games," Sports Culture Guide, vol. 6, pp. 18-23, 2019.

[32] Y. Du and B. Sun, "Research on the sustainable development of winter Olympic games host area: a case study of whistler resort in Vancouver," Sports Culture Guide, vol. 2, pp. 23-28, 2018.

[33] M. Chen, "Basic countermeasures for implementing national fitness program in developed and underdeveloped cities," Journal of Physical Education, vol. 4, pp. 24-26, 2000.

[34] L. Luo, "On the development of leisure sports industry in China from the interactive relationship between industry and culture," Journal of Beijing Sport University, vol. 12, pp. 1645-1647, 2006.

[35] J. Yu and X. Zuo, "A comparative study of leisure sports thought between China and west," Sports Culture Guide, vol. 6, pp. 68-69, 2008.

[36] L. Burney, Sports Management: Fundamentals and Applications, East China Normal University Press, Shanghai, China, 4th edition, 2009.

[37] Q. Zuo, Z. Huang, J. Su et al., Sports Management, Beijing Normal University Press, Beijing, China, 2010.

[38] Lu Yuan, "Institutional cost of the current management system of competitive sports in China," Journal of Physical Education, vol. 17, no. 3, pp. 7-12, 2010.

[39] Y. Zhu, M. Su, B. Dai, and H. Huang, "Research on competitive advantage of Chinese competitive sports," Sports Culture Guide, vol. 2, pp. 31-35, 2010.

[40] X. Han, H. Shen, and B. Zheng, "Research on the development of Chinese competitive sports in 60 years after the founding of the People's Republic of China," Sports Culture Guide, vol. 8, pp. 55-57, 2009.

[41] B. Huang, "Theory of factors in the development of competitive sports system in China," Journal of Sport Culture Tribune, vol. 5, pp. 5-8, 2015.

[42] T. Wen, "Mass sports development strategy research in China," Journal of Sport Culture Tribune, vol. 9, pp. 4-7, 2010.

[43] H. Dong and X. Fang, "Consideration on the benefit coordination between mass sports and competitive sports," Sports Culture Guide, vol. 8, pp. 16-24, 2009.

[44] Z. Jin-guo, "Social stratification and community sports development," Sports Culture Guide, vol. 9, pp. 34-37, 2011. 
[45] Y. Liu, W. Tang, J. He, and H. Pan, "Research on sports rights of disabled persons in China," Sports Culture Guide, vol. 3, pp. 17-20, 2010.

[46] Y. Liu, "De-structuring and reconstruction of public service system of sports for the elderly in China," Sports Culture Guide, vol. 2, pp. 5-8, 2014.

[47] Z. He, "College physical education reform and college students physical education ability cultivation," Journal of Wuhan Institute of Physical Education, vol. 3, pp. 110-112, 2000.

[48] B. Gao, M. Xu, R. Li, and B. Wang, "The reform of physical education teaching evaluation in colleges and universities," Journal of Physical Education, vol. 6, pp. 77-80, 2003.

[49] X. Xie and J. Mao, "Thoughts on the path of sports reform in China," Sports Culture Guide, vol. 3, pp. 5-7, 2009.

[50] M. Jing, "On the research object and the nature of the discipline of physical education and training," Sports and Science, vol. 33, no. 5, pp. 104-107, 2012.

[51] L. Cui, "On national traditional fitness sports and national fitness sports," Journal of Shandong Institute of Physical Education, vol. 4, pp. 44-50, 1998.

[52] T. Fan and D. Yu, "The current situation, problems and countermeasures of traditional martial arts inheritance based on the perspective of intangible cultural heritage," Journal of Nanjing Sports College (Social Science Edition), vol. 29, no. 1, pp. 27-31, 2015.

[53] J. Li and Z. Guo, "Regional martial arts heritage and international dissemination of Chinese martial arts: history and reality of martial arts culture in the Lingnan Pearl River Delta," Journal of Wuhan Institute of Physical Education, vol. 44, no. 3, pp. 56-60, 2010.

[54] X. Liu, W. Mao, and Y. Bai, "The difficulty of martial arts entering Olympic Games," Journal of Shandong Institute of Physical Education, vol. 2, pp. 31-32, 2005.

[55] H. Hong and L. Zhang, "Reflections on some theoretical issues of martial arts entering in the Olympic Games," Journal of Sports Culture, vol. 9, pp. 49-51, 2007.

[56] Lu Yuan, "Discipline integration and research frontier of sports humanities and sociology," Journal of Physical Education, vol. 1, pp. 4-7, 2005.

[57] L. Y. Zhen, Sports Sociology, Page Higher Education Press, Beijing, China, 3rd edition, 2010.

[58] Z. Yan, Z. He, and X. Li, "Research on the training path of reserve talents in football power," Sports Culture Guide, vol. 8, pp. 26-28, 2007.

[59] R. Liu and L. Pang, "Research on the training of reserve talents in competitive sports in China," China Sports Science and Technology, vol. 53, no. 4, pp. 42-47, 2017.

[60] Jinyu, S. Pan et al., "Current situation and countermeasure on cultivation of reserve talents of competitive sports in China," And Sports and Science, vol. 5, pp. 82-86, 2006.

[61] Y. Huang, Journal of Guangzhou Institute of Physical Education, vol. 36, no. 3, pp. 121-124, 2016.

[62] P. Zhou, R. Yu, and L. Dong, "Reform of physical education teaching content in colleges and universities," Physical Culture and History, vol. 6, p. 44, 2000.

[63] W. Xing, "Analysis on the current situation and development trend of mass sports research in China," Martial Arts Research, vol. 4, no. 2, pp. 137-147, 2019.

[64] R. Li and N. Liu, "Theoretical framework and logical path: research on high-quality development of sports industry in China," Journal of Tianjin Institute of Physical Education, vol. 35, no. 6, pp. 651-657, 2020.
[65] H. Guo and B. Ren, "High-quality development of Chinese sports industry in the new era: logical generation and path selection," Journal of Xi'an Institute of Physical Education, vol. 37, no. 3, pp. 291-297, 2020. 